Artículo Original/ Original Article

\title{
Impacto de la estrategia de entrega de frutas con educación nutricional sobre el estado nutricional y consumo frutas en escolares de la escuela pública Héroes Luqueños de la comunidad de Jukyry Luque, Paraguay
}

\author{
*Analía Núñez, Carolina Collante, María Isabel López, Clara Galeano \\ ${ }^{1}$ Universidad del Pacifico, Facultad de Ciencias Médicas, Carrera de Nutrición. Paraguay
}

Cómo referenciar este artículo/

How to reference this article:

\begin{abstract}
Núñez A, Collante $C$, López MI, Galeano $C$. Impacto de la estrategia de entrega de frutas con educación nutricional sobre el estado nutricional y consumo frutas en escolares de la Escuela pública héroes luqueños de la comunidad de Jukyry Luque, Paraguay. Mem. Inst. Investig. Cienc. Salud. 2019; 17(3): 55-62
\end{abstract}

\section{RE S U M E N}

En Paraguay, más del $50 \%$ de la población adulta presenta sobrepeso y obesidad, lo cual a su vez es un detonante para la génesis de otras afecciones como la diabetes y las enfermedades cardiovasculares. Hacer hincapié en la prevención es importante para disminuir la incidencia de las enfermedades crónicas no trasmisibles. Es importante desarrollar intervenciones que incidan en la formación de hábitos alimentarios saludables en niños y adolescentes de manera a que contribuyan a la prevención de enfermedades. El objetivo del estudio fue evaluar el impacto de la entrega de frutas con educación nutricional sobre el estado nutricional y consumo de frutas, además de evaluar la aceptabilidad de la intervención en adolescentes del séptimo grado de la Escuela Héroes Luqueños. Estudio cuasi-experimental en el que se realizó la evaluación de una intervención educativa que consistió en talleres sobre la importancia de consumir frutas para alumnos, docentes y padres con posterior entrega de frutas. Se realizó la evaluación pre y post intervención del estado nutricional y del conocimiento sobre consumo de frutas. Se hallaron diferencias significativas $(p<0,05)$ entre antes y después de la intervención en el promedio de circunferencia de cintura de los adolescentes, no así los indicadores IMC y talla. La entrega de frutas tuvo gran aceptación entre los adolescentes que participaron del estudio, por lo que se recomienda seguir con este tipo de intervenciones.

Palabras clave: educación nutricional, alimentación escolar, obesidad.

\section{Impact of fruit delivery with nutritional education on nutritional status and fruit consumption in school children of the Héroes Luqueños public school of the community of Jukyry Luque, Paraguay}

\section{A B S T R A C T}

In Paraguay, more than $50 \%$ of the adult population is overweight and obese, which in turn it is a trigger for the genesis of other conditions such as diabetes and cardiovascular diseases. Emphasis on prevention is important to reduce the incidence of noncommunicable chronic diseases. It is important to perform interventions that affects the formation of healthy eating habits in children and adolescents in order to contribute in the

Fecha de recepción: abril 2019. Fecha de aceptación: julio 2019

*Autor correspondiente: Analía Núñez. Universidad del Pacifico, Facultad de Ciencias Médicas, Carrera de Nutrición. Paraguay

Email: nutricionanalianunez@gmail.com 
Nuñez, et al Impacto de la estrategia de entrega de frutas con educación nutricional...

prevention of diseases. The objective of the study was to evaluate the impact of the delivery of fruits with nutritional education on the nutritional status and fruit consumption, in addition to evaluating the acceptability of the intervention in adolescents of the seventh grade of the Héroes Luqueños School. Quasi-experimental study in which the evaluation of an educational intervention was carried out, which consisted of workshops on the importance of consuming fruits for students, teachers and parents with subsequent fruit delivery. The pre and post intervention evaluation of nutritional status and knowledge about fruit consumption was carried out. Significant difference $(p<0.05)$ between pre and post intervention in the average waist circumference of the adolescents were found, but not in the BMI and height indicators. Fruit delivery was widely accepted among the adolescents who participated in the study, therefore it is recommended to continue with this type of intervention.

Keywords: Nutritional education, school feeding, obesity.

\section{INTRODUCCIÓN}

Las enfermedades crónicas no transmisibles representan actualmente un gran problema para la salud pública, muchas de ellas son prevenibles a través de estrategias que pueden ser ejecutadas durante el ciclo vital. La obesidad, la diabetes y la hipertensión; entre otras, tienen su origen en la niñez y la adolescencia, donde los hábitos alimentarios juegan un rol esencial. Las estrategias de prevención deben tener inicio en la población joven, para así evitar que la misma se convierta en una población adulta enferma que dependa de asistencia crónica por parte del estado ${ }^{(1)}$.

En nuestro país más del $50 \%$ de la población adulta presenta sobrepeso y obesidad, lo cual a su vez es un detonante para la génesis de otras afecciones como la diabetes y las enfermedades cardiovasculares; que en muchas ocasiones no son diagnosticadas y tratadas a tiempo ocasionando complicaciones que incluyen la discapacidad física en población de edad productiva e inclusive la muerte ${ }^{(1)}$.

Hacer hincapié en la prevención se vuelve de vital importancia para disminuir la incidencia de las enfermedades crónicas no trasmisibles, sobre todo aquellas en las que los hábitos alimentarios tienen gran implicancia como factor de riesgo, asociándose además al sedentarismo y a los antecedentes familiares.

Paraguay cuenta con una Estrategia Nacional para la Prevención y Control de la Obesidad (2015-2025) enmarcada en el Plan Nacional de Acción para la Prevención de ECNT 20142024, que tiene como propósito disminuir la obesidad de la población paraguaya en los próximos 10 años; en el contempla 4 áreas de acción, una de ellas es el Marco regulatorio, fiscalización y políticas que favorecen a la implementación de estrategias de prevención de obesidad, la cual busca implementar políticas para mejorar el acceso a alimentos saludables y tiene entre una de sus líneas de acción la regulación de la promoción, venta y publicidad de alimentos no saludables en instituciones educativas ${ }^{(2)}$.

Las acciones de esta investigación aportan a la Estrategia Nacional contra la Obesidad, específicamente al área de la Promoción de la Salud y prevención del sobrepeso y la obesidad a través de la entrega de fruta y educación nutricional dirigida a los alumnos que participaron $^{(2)}$.

La obesidad es definida comúnmente como una condición corporal caracterizada por la acumulación excesiva de grasa en el tejido adiposo, bajo la piel y en el interior de ciertos órganos, así como en los músculos. La acumulación de grasa, cuya capacidad energética es dos veces superior a la de las proteínas o carbohidratos, es una forma de almacenamiento energético para necesidades futuras ${ }^{(3)}$.

La obesidad se encuentra entre las enfermedades crónicas no transmisibles que representan actualmente un gran problema para la salud pública, muchas de ellas son prevenibles a través de estrategias que pueden ser ejecutadas durante el ciclo vital.

Para muchos países, los costos directos e indirectos que genera la atención de la obesidad son enormes. Por una parte, la obesidad al igual que otros padecimientos crónicodegenerativos, genera al paciente y su familia elevados costos de atención durante períodos largos de tratamiento. La necesidad de una dieta especial, -misma que frecuentemente resulta más costosa que la alimentación a la que muchos pacientes tienen acceso- y de la realización de actividad física -que además de tiempo para su realización implica también 
Nuñez, et al Impacto de la estrategia de entrega de frutas con educación nutricional...

otros gastos- se traducen junto con la compra de medicamentos, en una significativa carga a la economía familiar ${ }^{(3)}$.

Por otra parte, a nivel de las instituciones públicas y privadas de salud, la atención médica de estos pacientes es costosa y de largo plazo, pues tiene implícitas citas médicas frecuentes y exámenes médicos diversos para evaluar la evolución del problema, así como de sus enfermedades asociadas ${ }^{(3)}$.

Datos sobre el consumo de frutas de parte de los adolescentes, a nivel nacional se pueden encontrar en el informe de la Encuesta Nacional de Factores de Riesgo para Enfermedades No Transmisibles ${ }^{(4)}$ que indica que los estudiantes encuestados que declararon no haber consumido frutas llega al $7,9 \%$, refiere en la misma que no es un alto porcentaje, pero solo se refiere al consumo y no a la cantidad.

Según la Organización Mundial de la Salud, las frutas y las verduras son componentes esenciales de una dieta saludable, y un consumo diario suficiente podría contribuir a la prevención de enfermedades importantes ${ }^{(1)}$. Las recomendaciones internacionales son de $400 \mathrm{~g}$ diarios para prevenir enfermedades crónicas y mitigar las carencias de micronutrientes. Un consumo suficiente de frutas y verduras podría salvar hasta 1,7 millones de vidas cada año. La ingesta insuficiente de frutas y verduras es uno de los 10 factores principales de riesgo de mortalidad a escala mundial ${ }^{(1)}$. El consumo de frutas y verduras variadas garantiza un consumo suficiente de la mayoría de los micronutrientes, de fibra dietética y de una serie de sustancias no nutrientes esenciales. Además, el aumento del consumo de frutas y verduras puede ayudar a desplazar los alimentos ricos en grasas saturadas, azúcares o sal y de esa manera contribuir al estado nutricional adecuado y a una circunferencia de cintura adecuada.

El objetivo del estudio fue evaluar el impacto de la educación nutricional en el aumento del consumo de frutas y sobre el estado nutricional, además de evaluar la aceptabilidad de la intervención en adolescentes del 7 mo grado de la Escuela Héroes Luqueños.

\section{MATERIALES Y MÉTODOS}

Diseño del estudio: Observacional, descriptivo, cuasi experimental, longitudinal llevado a cabo en la Escuela Héroes Luqueños - Séptima compañía Jukyry - Luque. Departamento Central. Paraguay. Los sujetos fueron seleccionados según la lista oficial vigente al año lectivo para el 70 A y 70 B de la Escuela Pública Héroes Luqueños.

Criterios de Inclusión: Adolescentes de ambos sexos matriculados en el séptimo grado $A$ y B de la Escuela Héroes Luqueños - Séptima compañía Jukyry - Luque, Departamento Central, Paraguay y cuyos padres firmaron el consentimiento informado.

Criterios de exclusión: Adolescentes cuyos padres no firmaron el consentimiento informado, o los estudiantes se negaron a participar del estudio o con capacidades diferentes.

Talleres educativos: se realizaron talleres educativos destinados a los alumnos y a sus padres y al plantel de docentes.

Talleres educativos dirigidos a los alumnos: fueron realizados talleres de elaboración de aperitivos con frutas a fin de que los alumnos pudieran conocer otras formas de consumir frutas, fue una actividad dinámica donde se utilizaron frutas e insumos para el desempeño de los alumnos, se incluyeron distintos tipos de frutas y se otorgaron las indicaciones que cada alumno podría utilizar la creatividad que sugiera la combinación de verduras utilizando palitos y vasitos de plástico.

Talleres educativos dirigido a los padres de los alumnos: se desarrollaron temas de alimentación saludable y se priorizó la información sobre consumo de frutas y verduras y los beneficios del mismo.

Talleres educativos dirigido a plantel de docentes: se desarrollaron temas de las Guías Alimentarias del Paraguay de la familia a través de materiales educativos individuales y grupales, en una primera oportunidad el taller fue enfocado a una alimentación saludable y estableciendo como prioridad la alimentación en el hogar y el consumo de frutas y verduras y en el segundo taller se focalizaron las enfermedades crónicas no transmisibles.

Entrega de frutas: En una primera etapa se recibieron las frutas, se utilizó el protocolo de manipulación, se distribuyó por turno (mañana y tarde) los días lunes, miércoles y viernes, 
Nuñez, et al Impacto de la estrategia de entrega de frutas con educación nutricional...

con el apoyo del docente de la asignatura de los alumnos, la fruta fue entregada antes del receso en horarios de clase.

Las variables analizadas fueron la edad, estado nutricional por los indicadores antropométricos IMC, peso, talla para la edad, puntaje zeta, y circunferencia de cintura, además de los hábitos de consumo de frutas, número de veces que consumió fruta en el día.

El peso se midió con balanza digital de pie de la marca SECA utilizando la técnica recomendada por el Manual básico de Antropometría del Instituto Nacional de Alimentación y Nutrición - INAN. La talla se midió considerando el plano de Frankfurt y utilizando la técnica recomendada por el Manual básico de Antropometría del Instituto Nacional de Alimentación y Nutrición - INAN. Con tallímetro portátil de la marca SECA. El Índice de Masa Corporal: corresponde a la relación del peso entre la estatura en metros cuadrados. IMC $=$ Peso/talla ${ }^{2}$ (en metros).

El puntaje Z: corresponde a la desviación estándar que presenta el IMC en referencia a la mediana del patrón de crecimiento de la OMS para niños y adolescentes.

La circunferencia de cintura se midió y se evaluó considerando la técnica y la referencia NHANES 1988 recomendada por el Manual básico de Antropometría del Instituto Nacional de Alimentación y Nutrición - INAN. Se utilizó una cinta métrica metálica inextensible.

Estado nutricional: una vez obtenido el IMC, la talla y la edad de los sujetos se evaluó según los patrones de crecimiento para niños de 5 a 19 años de la Organización Mundial de la Salud OMS 2007. Se clasificaron según indicador IMC/Edad en: sin desnutrición, riesgo de desnutrición, desnutrición, sobrepeso y obesidad y según indicador Talla/Edad en: adecuado, riesgo de talla baja, talla baja y sin talla baja.

Encuesta de Hábitos alimentarios y satisfacción con la intervención: Se aplicaron dos test, denominados "pre test" y "post test", el primero consistió en preguntas sobre hábitos y actitudes comportamentales sobre el consumo de frutas, en el segundo se detallaron preguntas sobre la percepción del programa de entrega de frutas y la satisfacción con la misma.

\section{RESULTADOS}

En el estudio basal antes de realizar la entrega de frutas y los talleres educativos se evaluaron 37 adolescentes ( 24 mujeres y 24 varones), de los cuales 26 pertenecían al turno mañana y 11 al turno tarde. La mediana de edad fue de 12 años. Según el indicador Índice de Masa Corporal $\left(\mathrm{IMC}=\mathrm{Peso} / \mathrm{T}^{2}\right.$ ) para la edad el estado nutricional de los escolares fue malnutrición por exceso casi en la mitad de la muestra $(46 \% ; n=17)$, observándose sobrepeso en un $29,7 \%(n=11)$ y obesidad en un $16,2 \%(n=6)$. La malnutrición por déficit solo se encontró en un participante $(2,70 \% 1)$. La evaluación nutricional según el indicador Talla (estatura en centímetros) para la edad mostró un $29 \%$ que presentaba déficit en su crecimiento (desnutrición crónica), de los cuales $27,03 \%(n=10)$ presentó riesgo de talla baja y $2,70 \%(n=1)$ talla baja. La evaluación de la circunferencia de cintura arrojó un $8,11 \%(n=3)$ de los escolares con circunferencia de cintura aumentada (Tabla 1$)$.

La evaluación antropométrica posterior a la entrega de frutas fue realizada a 14 sujetos; 10 del turno mañana y 4 del turno tarde. Debido al retraso en el inicio de las actividades, y considerando que muchos de los adolescentes ya no acudieron a las clases durante el mes de noviembre no fue posible acceder a un mayor número de participantes. El $35,71 \%(n=5)$ de los evaluados presentó sobrepeso y un 7,14\% $(n=1)$ riesgo de desnutrición, ninguno presentó obesidad. En cuanto a la talla, un $14,29 \%(n=2)$ presentó riesgo de talla baja (riesgo de desnutrición crónica). Con respecto a la circunferencia de cintura; el $100 \%$ $(n=14)$ presentó en el rango adecuado (Tabla 1$)$.

Tabla 1: Estado nutricional de los escolares antes y después de la entrega de frutas

\begin{tabular}{lrr}
\hline Variables & $\begin{array}{r}\text { Antes } \\
\mathbf{n = 3 7}\end{array}$ & $\begin{array}{r}\text { Después } \\
\mathbf{n = 1 4}\end{array}$ \\
\hline Estado nutricional según indicador IMC/Edad & $19(51,35)$ & $8(57,1)$ \\
Sin desnutrición & $1(2,70)$ & $1(7,14)$ \\
Riesgo de desnutrición & $11(29,73)$ & $5(35,71)$ \\
Sobrepeso & $6(16,22)$ & - \\
Obesidad & & \\
\hline
\end{tabular}


Nuñez, et al Impacto de la estrategia de entrega de frutas con educación nutricional...

\begin{tabular}{lrr}
\hline Estado Nutricional según indicador Talla/Edad & & \\
Sin talla baja & $26(70,27)$ & $12(85,71)$ \\
Riesgo de talla baja & $10(27,03)$ & $2(14,29)$ \\
Talla baja & $1(2,70)$ & - \\
Circunferencia de cintura & $\mathbf{N}$ & \\
Adecuada & $34(91,89)$ & $14(100,00 \%)$ \\
Aumentada & $3(8,11)$ & - \\
\hline
\end{tabular}

En las Tabla 2 se observan los resultados de las encuestas realizadas a los adolescentes antes y después de la entrega de frutas. En el "pre test" se encontró que el $84,6 \%$ de los adolescentes declararon consumir "toda la fruta". La pregunta "tu familia come frutas cuando estás con ellos" es interpretada como la formación de hábitos, ya que la familia es la protagonista como formadora de los mismos porque en la casa se ofrecen alimentos según la disponibilidad de los mismos pero también de acuerdo a la preferencia y conocimientos relacionados a los alimentos de los propios padres o encargados, en éste caso el resultado fue que "a veces" se comparte en familia el consumo de frutas siendo este dato en su mayoría $(65,3 \%)$.

Los adolescentes respondieron que no traían frutas en el recreo porque traían el dinero $(82,3 \%)$, u otro alimento/comida $(17,3 \%)$, esto se considera una oportunidad pérdida ya que los adolescentes adquirien los alimentos disponibles en la cantina, y la misma no ofrece frutas o preparaciones que incluyan frutas. La mayoría $(92,3 \%)$ contestó que están dispuestos a ingerir nuevas frutas.

Los resultados del "post test" mostraron aumento en el porcentaje de consumo de toda la fruta y de la fruta entera, sin embargo, no hubo aumento en la frecuencia del consumo con la familia o cuando los amigos comen otro alimento. Se incluyeron preguntas para evaluar la satisfacción de los escolares con la entrega de frutas lo que resultó que los adolescentes estuvieron $100 \%$ conformes y el $60 \%$ respondió que no cambiaría las frutas entregadas.

Tabla 2: Resultados de las encuestas realizadas antes y después de la entrega de frutas.

\begin{tabular}{|c|c|c|}
\hline Items & Antes $\mathbf{n = 2 6}$ & $\begin{array}{l}\text { Después } \\
n=15\end{array}$ \\
\hline \multicolumn{3}{|c|}{ ¿Cuándo consumes frutas comes toda la fruta o la mitad? } \\
\hline Toda la fruta & $22(84,62)$ & $14(93,33)$ \\
\hline La mitad de la fruta & $4(15,38)$ & $1(6,67)$ \\
\hline \multicolumn{3}{|c|}{ ¿Tu familia come frutas cuando estas con ellos? } \\
\hline Siempre & $8(30,77)$ & $5(33,33)$ \\
\hline A veces & $17(65,38)$ & $9(60)$ \\
\hline Nunca & $1(3,85)$ & $1(6,67)$ \\
\hline \multicolumn{3}{|c|}{ ¿Comes frutas cuando tus amigos comen otro alimento? } \\
\hline Siempre & $7(26,92)$ & $4(26,67)$ \\
\hline A veces & $10(38,46)$ & $5(33,3)$ \\
\hline Nunca & $9(34,62)$ & $6(40)$ \\
\hline \multicolumn{3}{|l|}{ ¿De qué forma comes las frutas? } \\
\hline Entera & $16(61,54)$ & $12(80)$ \\
\hline Jugo natural con azúcar & $4(15,38)$ & - \\
\hline \multicolumn{3}{|c|}{ ¿Motivo por el cual no trae fruta para el recreo? } \\
\hline Traen dinero & $14(82,3)$ & - \\
\hline Traen otra comida & $3(17,3)$ & - \\
\hline \multicolumn{3}{|c|}{ ¿Estarías dispuesto a probar frutas nuevas? } \\
\hline $\mathrm{Si}$ & $24(92,31)$ & - \\
\hline No & $2(7,69)$ & - \\
\hline \multicolumn{3}{|c|}{ ¿Qué te pareció haber recibido frutas en la media mañana? } \\
\hline \multicolumn{3}{|c|}{ ¿Qué mejoraría o cambiarías del programa de entrega? } \\
\hline No cambiaría la variedad de frutas & - & $9(60)$ \\
\hline Entregar frutas con mayor variedad & & $6(40)$ \\
\hline
\end{tabular}


Nuñez, et al Impacto de la estrategia de entrega de frutas con educación nutricional...

\section{DISCUSIÓN}

Según los datos proveídos a través de la primera Encuesta Mundial de Salud Escolar (EMSE) llevada a cabo en Paraguay en el año 2017, el estado nutricional de los adolescentes (octavo y noveno grados) se presentó de la siguiente forma; $2,0 \%$ de bajo peso, $25,3 \%$ de sobrepeso y $7,4 \%$ de obesidad. Obteniendo un porcentaje de malnutrición por exceso del $32,7 \%{ }^{(11)}$. Datos similares se han hallado en la presente investigación, considerando los resultados de la evaluación nutricional aplicada a 37 adolescentes del séptimo grado de la Escuela pública "Héroes Luqueños" de la comunidad Jukyry de Luque; antes de la entrega de frutas. La malnutrición por exceso se observa casi en la mitad de la muestra (46\%), observándose obesidad en un $16,2 \%$ y sobrepeso en un $29,7 \%$. La malnutrición por exceso ha cobrado relevancia en los últimos tiempos, considerando que su aparición temprana (niñez y adolescencia), implica un factor de riesgo importante para el desarrollo de enfermedades crónicas como las cardiovasculares y metabólicas como la diabetes mellitus tipo 2. Así mismo su frecuencia en la población adulta es preocupante para la salud pública ${ }^{(1)}$.

En ese contexto el consumo de frutas ha sido encarado como un hábito saludable que pudiera contribuir a la reducción de la malnutrición, inclusive la malnutrición derivada de la falta de vitaminas ${ }^{(4)}$. Este proyecto de intervención tuvo gran aceptación en los adolescentes, quienes recibieron frutas como media mañana tres veces por semana.

No se encontraron diferencias significativas en el estado nutricional (índice de masa corporal y talla para la edad) de los adolescentes antes y después de la entrega de frutas, sin embargo, se hallaron diferencias significativas en el promedio de circunferencia de cintura de los adolescentes, antes y después de la entrega de frutas $(p<0,05)$. Antes de la intervención se encontraba en $91.9 \%$ adecuada y $8.11 \%$ aumentada, luego de la intervención adecuada en $100 \%$, aunque actualmente no existen consensos sobre cuáles son los puntos de corte recomendados de circunferencia de cintura para adolescentes, este estudio utilizo la norma técnica esgrimida por el Instituto Nacional de Alimentación y Nutrición (INAN) en su manual básico de antropometría, donde una circunferencia de cintura por encima del Pc 90 es considerada como "aumentada", en consideración al riesgo cardiovascular.

En este estudio se observó que los adolescentes redujeron 2 centímetros en promedio de circunferencia de cintura luego de la entrega de frutas $(p=0.008)$. En un estudio similar realizado en Chile (Universidad de Chile; Programa 5 al día) por González C. et al., 2014, no se observaron diferencias significativas en el estado nutricional según IMC (Índice de Masa Corporal) y Talla para la Edad de los niños y adolescentes antes y después de la entrega de frutas, en el mismo no se tuvo en cuenta la medición de la circunferencia de cintura de los sujetos $^{(16)}$.

Los hallazgos del estudio FLASHE 2018 (Family Life, Activity, Sun, Health, and Eating) muestran que la conducta de los padres en cuanto a sus preferencias alimentarias, puede impactar en gran medida el hábito del consumo de frutas y vegetales de los adolescentes $^{(17)}$. En el presente estudio la falta de adherencia de los padres y/o encargados de los adolescentes significó un obstáculo para alcanzar el número de muestra requerido como así también la participación de los mismos en los talleres educativos.

En un estudio llevado a cabo por Quizán Plata et al., 2012 donde se midió la efectividad de un programa de alimentación saludable en escuelas públicas de Sonora, se vio que las instituciones que fueron intervenidas con campañas educativas, aumentaron su consumo de frutas durante el recreo, en cambio aquellas donde no se intervino presentaron mayor riesgo de consumir alimentos no saludables, la intervención tuvo un tiempo de 6 meses ${ }^{(18)}$.

Por nuestra parte el tiempo de intervención fue escaso, por lo cual la realización de los talleres educativos no se pudo concretar en su totalidad, de forma a obtener datos a través de los cuales se pueda medir el impacto de la intervención educativa. No obstante, en los talleres educativos realizados se pudo exponer a los adolescentes las diversas formas del consumo de frutas, una de ellas, en jugos, ya que los mismos son bien aceptados por los niños y adolescentes y podrían suplir el consumo de bebidas gaseosas durante los recreos, además de estar demostrado que también pueden ser fuente de vitaminas y otros nutrientes ${ }^{(19)}$. 
En un estudio llevado a cabo en el estado de Pelotas (Brasil), se realizó el seguimiento de una cohorte de adolescentes por 3 años (de 15 a 18 años de edad), evaluando su consumo de frutas y vegetales, en el mismo se pudo observar que el consumo de este grupo de alimentos fue moderadamente estable, sobre todo en aquellos adolescentes con mayor poder adquisitivo ${ }^{(20)}$.

Considerando esos datos, es necesario que las estrategias para la promoción del consumo de frutas vayan acompañadas por medidas que faciliten el acceso a las mismas, ya que, en muchas ocasiones, cuando la fruta no es parte de la merienda escolar, no es consumida por su mayor costo en comparación a otros alimentos no saludables. La entrega de frutas en el presente estudio tuvo gran aceptación entre los adolescentes que participaron del mismo.

Financiación: Este estudio de investigación se realizó dentro del marco de Fondos Concursables en Investigación de la Universidad del Pacífico en su convocatoria 2018.

\section{REFERENCIAS BIBLIOGRÁFICAS}

1. Organización Mundial de la Salud. Perfil de las enfermedades crónicas por países. Informe de la Organización Mundial de la Salud 2018. Ginebra: OMS; 2011.

2. Organización Mundial de la Salud. Datos y cifras sobre las enfermedades crónicas no transmisibles. Reporte técnico de la Organización Mundial de la Salud. Disponible en https://www. who.int/features/factfiles/no ncommunicable_diseases/facts/es/

3. Organización Panamericana de la Salud. Plan de acción para la prevención de la obesidad en la niñez y la adolescencia. Organización Panamericana de la Salud. Washington DC; 2014. Disponible en https://www.paho.org/hq/dmdocuments/2 015/Obesity-Plan-Of-Action-Child-Spa2015.pdf

4. OMS. Fomento del consumo mundial de frutas y verduras (Internet). WHO. (citado 20 de agosto de 2019). Disponible en: https://www.who.int/dietphysicalactivity/f ruit/es/.

5. Hartley L, Igbinedion E, Holmes J, Flowers $\mathrm{N}$, Thorogood $M$, Clarke $A$, et al. Increased consumption of fruit and vegetables for the primary prevention of cardiovascular diseases. Cochrane Database of Systematic Reviews 2013, Issue 6. Art. No.: CD009874. DOI: 10.1002/14651858.CD009874.pub2.

6. Ministerio de Salud de la Argentina. Educación alimentaria y nutricional - Libro para el docente. Buenos Aires: FAO; 2009.

7. Martin A, Booth JN, Laird Y, Sproule J, Reilly JJ, Saunders DH. Physical activity, diet and other behavioral interventions for improving cognition and school achievement in children and adolescents with obesity or overweight. Cochrane Database of Systematic Reviews 2018; (3). Art. No.: CD009728. DOI: 10.1002/14651858.CD009728.pub4.

8. Ministerio de Salud Pública y Bienestar Social. Primera Encuesta Nacional de
Factores de Riesgo para Enfermedades No Transmisibles. Asunción: MSPBS; 2011.

9. Ministerio de Salud Pública y Bienestar Social. Dirección de Vigilancia de las Enfermedades Crónicas no transmisibles. Estrategia Nacional para la prevención y control de la Obesidad 2015-2025. Asunción: MSPBS; 2015.

10. Ministerio de Educación y Cultura. Resolución $N^{\circ}$ 16.264. procedimiento y monitoreo para la explotación y el usufructo de las cantinas de las instituciones educativas. Asunción: MEC; 2013.

11. Ministerio de Salud Pública y Bienestar Social. Encuesta Global de Salud Escolar GSHS. Asunción: OPS/OMS. ; 2017. Disponible https://www.mspbs.gov.py/dependencias/ portal/adjunto/e1e76b-

EncuestaGlobalGSHSvistaporpagina.pdf

12. Ministerio de Salud Pública y Bienestar Social. Escuelas Saludables - Guía de Gestión. Asunción: MSPBS; 2014.

13. Langford $\mathrm{R}$, Bonell $\mathrm{CP}$, Jones $\mathrm{HE}$, Pouliou $T$, Murphy SM, Waters E, et al. The WHO Health Promoting School framework for improving the health and well-being of students and their academic achievement. Cochrane Database of Systematic Reviews 2014, Issue 4. Art. No.: CD008958. DOI: 10.1002/14651858.CD008958.pub2.

14. Waters E, de Silva-Sanigorski A, Burford BJ, Brown T, Campbell KJ, Gao $Y$, Armstrong $\mathrm{R}$, Prosser $\mathrm{L}$, Summerbell CD. Interventions for preventing obesity in children. Cochrane Database of Systematic Reviews 2011, Issue 12. Art. No.: CD001871. DOI: 10.1002/14651858.CD001871.pub3.

15. Wolfenden $L$, Nathan NK, Sutherland $R$, Yoong SL, Hodder RK, Wyse RJ, et al. Strategies for enhancing the implementation of school-based policies or practices targeting risk factors for chronic disease. Cochrane Database of Systematic 
Nuñez, et al Impacto de la estrategia de entrega de frutas con educación nutricional...

Reviews 2017, Issue. Art. No.: CD011677. DOI: $10.1002 / 14651858 . C D 011677 . p u b 2$.

16. González G CG, Zacarías H I, Domper R A, Fonseca $M L$, Lera $M$, Vio del $R F$. Evaluación de un programa de entrega de frutas con educación nutricional en escuelas públicas rurales de la Región Metropolitana, Chile. Rev. chil. nutr. (Internet). 2014 Sep. Disponible en: https://scielo.conicyt.cl/scielo.php?script= sci_arttext\&pid=S0717-

$75182014000300001 \&$ lng $=$ es.http://dx.do i.org/10.4067/S0717-

75182014000300001.

17. Parks CA, Blaser C, Smith TM, Calloway EE, Oh AY, Dwyer LA, et al. Correlates of fruit and vegetable intake among parents and adolescents: findings from the Family Life, Activity, Sun, Health, and Eating (FLASHE) study. Public Health Nutrition. Cambridge University Press; 2018; 21(11):2079-87.

18. Quizán Plata T, Barragán AC, Esparza Romero EJ, Orozco García ME, Espinoza López A, Bolaños Villar AV. Efectividad del programa Promoción de alimentación saludable en estudiantes de escuelas públicas del estado de Sonora. Estud Soc (revista en Internet). 2013 (citado 20 Ago 2019); 21(42): (aprox. 9p). Disponible en:

http://www.scielo.org.mx/scielo.php?scrip $\mathrm{t}=\mathrm{sci}$ _arttext\&pid=S018845572013000200008.

19. O'Neil CE, Nicklas TA, Zanovec M, Kleinman RE, Fulgoni VL. Fruit juice consumption is associated with improved nutrient adequacy in children and adolescents: the National Health and Nutrition Examination Survey (NHANES) 2003-2006. Public Health Nutrition. Cambridge University Press; 2012; 15 (10):1871-8.

20. Buffarini R, Muniz LC, Barros AJD, Araújo $C L$, Gonçalves $H$, Menezes $A M B$, et al. Stability and change in fruit and vegetable intake of Brazilian adolescents over a 3year period: 1993 Pelotas Birth Cohort. Public Health Nutrition. Cambridge University Press; 2016; 19(3):386-92. 